\title{
Holter Monitoring (24-h Electrocardiography) of Holstein Calves
}

\author{
Rebecca Bastos Pessoa, Camila Freitas Batista, Kamila Reis Santos, \\ Jéssyca Beraldi Bellinazzi, Alice Maria Melville Paiva Della Libera \& Maria Helena Matiko Akao Larsson
}

\begin{abstract}
Background: Twenty-four h electrocardiographic monitoring is a noninvasive method of assessing cardiac rhythm. Holter monitoring in farm animals can help assessing heart rate variability and its relationship with stress and production. Several authors have reviewed the normal cardiac rhythm of bovines, but there is little information on heart rhythm in calves. The goal of this research is to elucidate which cardiac rhythms may be considered physiological in Holstein calves, from 3 to 6 months old.
\end{abstract}

Materials, Methods \& Results: Twenty-four h ambulatory electrocardiography (Holter monitoring) was performed in 10 male Holstein calves, with ages ranging from 3 to 6 months old. The animals came from private farms in São Paulo state and were housed in a semi-open stall during the study. The animals had their hair clipped from the third to the fifth intercostal spaces on both sides of the chest, from the sternal region to the glenohumeral joint, and seven electrodes were positioned in a diagonal configuration. Decoding of the recordings was performed using specialized software and in order to minimize digital mistakes, all of the beats marked as ectopic by the software were manually revised by the authors. Mean heart rate was $83.2 \pm 11.06$ beats per $\min (\mathrm{bpm})$, maximum heart rate was $147.1 \pm 11.05 \mathrm{bpm}$, and minimum heart rate was $53.7 \pm 7.45 \mathrm{bpm}$. In $90 \%$ of the calves, normal sinus rhythm was predominant, whereas in $10 \%$, sinus arrhythmia (SA) was prevalent. Second degree atrioventricular blocks (AV blocks) were observed in $30 \%$ of the animals and supraventricular premature beats (SPB) were detected in 50\%. Holter monitoring was repeated after three months in three of the animals; two showed no arrhythmias at the second exam, and in the other, the frequency of AV blocks was decreased by 88.64\%.

Discussion: In the present study, the authors observed a relatively high incidence of arrhythmias in Holstein calves, although there is little information available in the literature for comparison. The use of Holter monitoring is much more sensitive in the assessment of arrhythmias than a standard electrocardiogram, since it records the cardiac rhythm for 24 to $48 \mathrm{~h}$, as opposed to only 2 to $3 \mathrm{~min}$. Therefore, it is possible that the arrhythmias found in the calves in the current study were physiological events otherwise unidentified by conventional electrocardiogram. The AV blocks observed in the animals of the present study were intermittent and apparently non-related to any particular event or situation, so it was not possible to demonstrate whether they disappeared after exercise. As for the supraventricular ectopic beats, they are frequent in older bovines, mainly in dairy cows, and are usually related to increased vagal tone, stress, hormonal changes, ectopic atrial focus, and peak lactation volume overload. The animals used in the present study were otherwise healthy, and did not demonstrate any clinical signs of gastrointestinal dysfunction or other disease that might have justified an increase in their vagal tone. In addition, the decrease in the number of rhythm abnormalities in the older calves that were submitted to a second Holter exam supports the hypothesis that supraventricular ectopic beats and second degree AV blocks of Mobitz type II may be normal in calves up to six months old.

Keywords: electrocardiography, veterinary cardiology, Holstein, calves, buiatrics. 


\section{INTRODUCTION}

Twenty-four h electrocardiographic monitoring is a noninvasive method of assessing cardiac rhythm. It is much more sensitive in the assessment of arrhythmias than a standard electrocardiogram (ECG), since it records the cardiac rhythm for 24 to $48 \mathrm{~h}$, as opposed to only 2 to $3 \mathrm{~min}$ [7]. Besides aiding the diagnosis of cardiac arrhythmias, it is also indicated to correlate clinical manifestations with periods of arrhythmia, assess the need for treatment of arrhythmias, evaluate the efficacy of antiarrhythmic therapy, and detect occult cardiomyopathies [7].

Most of the research on Holter monitoring in farm animals has aimed to assess heart rate variability and its relationship with stress and production [2]. Several authors have reviewed the normal cardiac rhythm of bovines, but there is little information on heart rhythm in calves.

There is disagreement in the literature as to whether sinus arrhythmia is rare in bovines, or if it is actually frequent $[4,10]$. There are reports of arrhythmia in Holstein dairy cows, which involve mainly atrial fibrillation [3,9]. There are also reports of atrioventricular blocks (AV blocks) and atrial and ventricular ectopic beats, but these are low in number [10]. Nevertheless, the small amount of research involving Holter monitoring in ruminants indicates that some types of arrhythmias may have been underestimated in this species, since most of the research has used conventional ECG. Therefore, the goal of this research is to elucidate which cardiac rhythms may be considered physiological in Holstein calves.

\section{MATERIALS AND METHODS}

Ten male Holstein calves were used in the present study. They came from private farms in São Paulo state, and during the experiment, they were aged from three to six months. The animals were housed in a semi-open stall at the Veterinary Hospital of Cattle and Small Ruminants of the School of Veterinary Medicine and Animal Science of the University of São Paulo. The animals were fed with concentrated food two times a day and ad libitum hay and water.

A full clinical examination was performed in each of the animals before the beginning of every recording. The animals had their hair clipped from the third to the fifth intercostal spaces on both sides of the chest, from the sternal region to the glenohumeral joint. Seven electrodes were positioned in a diagonal configu- ration, with three of them on the right side of the chest, and four on the left side. The wires and recorders were protected by one layer of elastic bandage and two layers of adhesive tape. The recorders used included analogical (Dynamis) ${ }^{1}$ and digital (DMS 300-6) ${ }^{2}$ devices.

Decoding of the recordings was performed using specialized software (CardioScan 10) ${ }^{2}$. In order to minimize digital mistakes, all of the beats marked as ectopic by the software were manually revised by the authors. Analysis of the results included a 24-h summary of medium, maximum, and minimum heart rates, predominant heart rhythm, number of ectopic beats per hour, and atrioventricular or sinus abnormalities. In order to estimate the mean time of bradycardia and tachycardia in each animal, the 24-h summary of heart rate was used, and rates above 100 beats per min were considered tachycardia, while those below 70 beats per min were considered bradycardia. Sinus arrhythmia was diagnosed when consecutive RR intervals differed by more than $10 \%$ in their length. Statistical analysis was made using Graph Pad Instat software ${ }^{3}$.

\section{RESULTS}

The results are summarized in Tables 1 and 2 . Calves 1, 2, and 9 are identified as 1a, 1b, 2a, 2b, 9a, and $9 \mathrm{~b}$ because each of them underwent the Holter exam twice, with an interval of approximately 3 months between the recordings. No ectopic beats were identified in calves 1 and 9 during the second recording, and the frequency of AV blocks was decreased by $88.64 \%$ in calf 2 . The estimated percentage of bradycardia and tachycardia in the recordings for each animal is summarized in Table 3.

\section{DISCUSSION}

Heart rhythm abnormalities in bovines are not rare, but their significance is not always clear [10]. In the present study, the authors observed a relatively high incidence of arrhythmias in Holstein calves, although there is little information available in the literature for comparison. Most of the reports regarding assessment of heart rhythm in bovines have been performed using conventional ECG $[6,10]$ and have focused mainly on heart rate variability [5]. The use of 24-h ambulatory ECG in the present study enabled the standardization of normal cardiac rhythms in Holstein calves as well as the identification of possibly underestimated arrhythmias in this population. 
R.B. Pessoa, C.F. Batista, K.R. Santos, et al. 2016. Holter Monitoring (24-h Electrocardiography) of Holstein Calves.

Table 1. Holter parameters of Holstein calves from three to six months old. São Paulo, 2015.

\begin{tabular}{|c|c|c|c|c|c|c|c|c|}
\hline \multirow[b]{2}{*}{$\begin{array}{c}\text { Calf } \\
\text { identification }\end{array}$} & \multicolumn{8}{|c|}{ Holter parameters } \\
\hline & $\mathrm{QRS} / \mathrm{min}$ & $\begin{array}{l}\text { Length of } \\
\text { recording } \\
(\min )\end{array}$ & Interf (\%) & HR mean & HR max & $\begin{array}{l}\text { HR } \\
\text { min }\end{array}$ & $\mathrm{HR}>100(\mathrm{~min})$ & $\mathrm{HR}<70(\mathrm{~min})$ \\
\hline 1 & 96.04 & 1398 & 0.42 & 96 & 140 & 64 & 440 & 0 \\
\hline 2 & 98.056 & 1406 & 0.18 & 98 & 160 & 67 & 540 & 0 \\
\hline 3 & 96.02 & 1372 & 1.71 & 96 & 151 & 57 & 715 & 0 \\
\hline 4 & 75.007 & 1410 & 1.89 & 75 & 157 & 52 & 35 & 565 \\
\hline 5 & 69.049 & 1302 & 0.89 & 69 & 144 & 43 & 5 & 125 \\
\hline 6 & 90.048 & 1313 & 0.16 & 90 & 144 & 54 & 300 & 55 \\
\hline 7 & 83.032 & 1277 & 4.46 & 83 & 122 & 55 & 0 & 185 \\
\hline 8 & 73.005 & 1440 & 0.23 & 73 & 144 & 48 & 20 & 470 \\
\hline 9 & 78.815 & 1262 & 0.53 & 72 & 155 & 48 & 60 & 500 \\
\hline 10 & 80.03 & 1390 & 0.23 & 80 & 154 & 49 & 20 & 345 \\
\hline Mean \pm SD & $83.91 \pm 10.5$ & $1357 \pm 62.79$ & $1.07 \pm 1.35$ & $83.20 \pm 11.06$ & $147.10 \pm 11.05$ & $53.70 \pm 7.45$ & $213.50 \pm 265.88$ & $224.50 \pm 225.59$ \\
\hline
\end{tabular}

$\overline{\mathrm{QRS}} / \mathrm{min}=$ number of QRS complexes per min of recording; Interf= interference; HR= heart rate; HR $>100=$ period of time HR was higher than 100 $\mathrm{bpm} ; \mathrm{HR}<70=$ period of time HR was lower than $70 \mathrm{bpm}$.

Table 2. Number of arrhythmias detected by the Holter exam in Holstein calves. São Paulo, 2015.

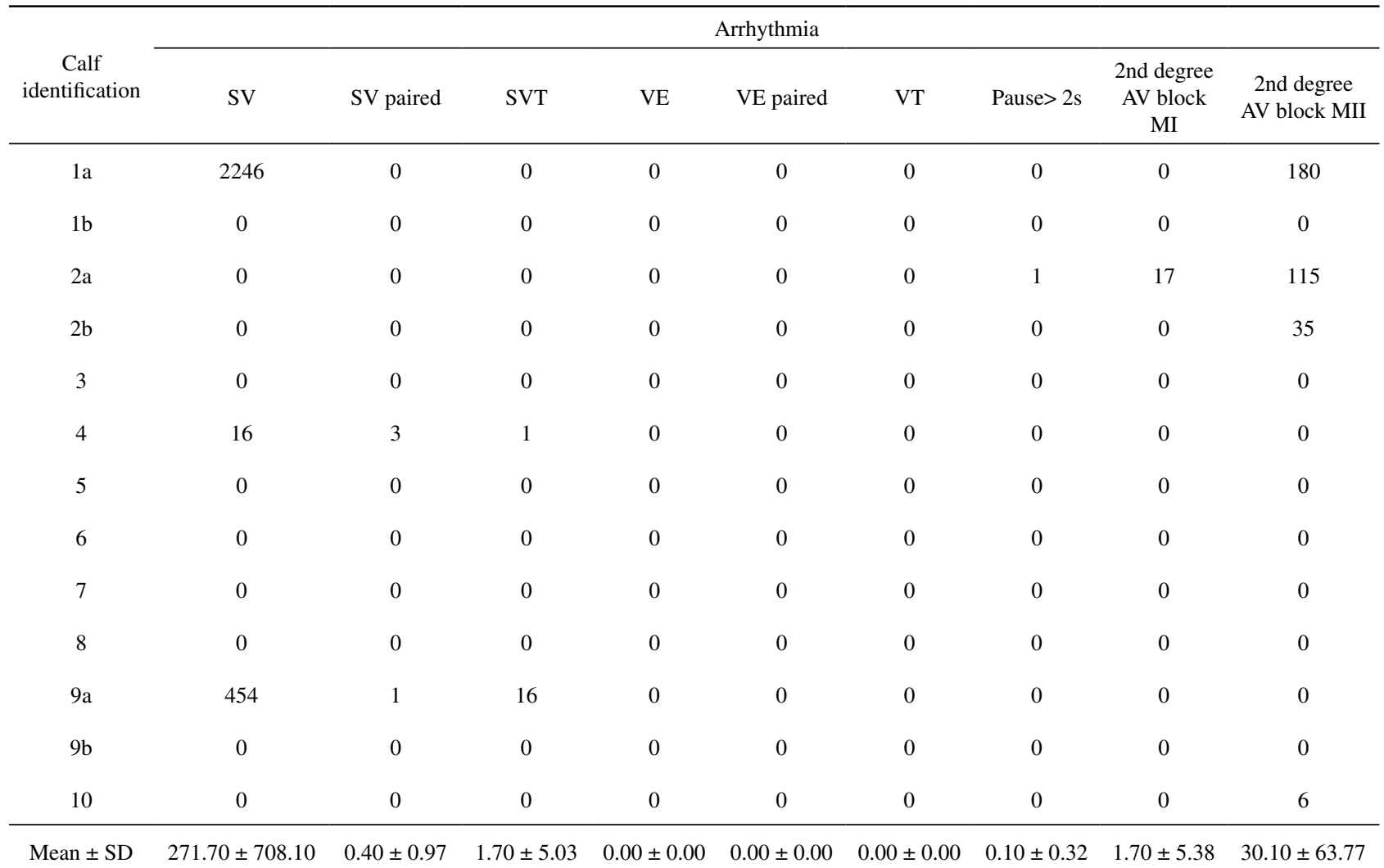

$\overline{\mathrm{SV}}=$ supraventricular ectopic beats; SV paired= paired supraventricular ectopic beats; $\mathrm{SVT}=$ supraventricular tachycardia; $\mathrm{VE}=$ ventricular ectopic beats; VE paired= paired ventricular ectopic beats; VT= ventricular tachycardia; $2^{\text {nd }}$ degree AV block MI= second degree atrioventricular block of Mobitz type I; $2^{\text {nd }}$ degree $\mathrm{AV}$ block MII= second degree atrioventricular block of Mobitz type II. 
Table 3. Percentage of time in bradycardia and tachycardia observed in Holstein calves. São Paulo, 2015.

\begin{tabular}{ccc}
\hline \multirow{2}{*}{ Calf identification } & \multicolumn{2}{c}{ Time (\%) } \\
\cline { 2 - 3 } & HR $>100$ bpm & HR $<70$ bpm \\
\hline 1 & 31.47 & 0 \\
2 & 38.40 & 0 \\
3 & 52.11 & 0 \\
4 & 2.48 & 40.07 \\
5 & 0.03 & 9.60 \\
6 & 22.84 & 4.18 \\
7 & 0 & 14.48 \\
8 & 1.38 & 32.63 \\
9 & 4.75 & 39.61 \\
10 & 1.43 & 24.82 \\
\hline
\end{tabular}

$\mathrm{AV}$ blocks are not as common in bovines as they are in horses [10], but they have been reported in this species by authors using conventional ECG $[6,10]$. In the present study, this conduction abnormality was observed in $30 \%$ of the animals, and second degree AV block was the most common type. In this type of block, electric conduction from the atria to the ventricle is temporarily interrupted, and isolated $\mathrm{P}$ waves are present on the tracing, although most of the electric impulses are conducted and generate normal QRS complexes. In horses, second degree AV block is considered physiological when it disappears after exercise, and is related to increased vagal tone [8]. The AV blocks observed in the animals of the present study were intermittent and apparently nonrelated to any particular event or situation, so it was not possible to demonstrate whether they disappeared after exercise. There are reports of second degree AV blocks in both healthy and sick cattle, but the majority of these were related to calves presenting diarrhea or plant poisoning [6,10]. While second degree AV blocks have been described in cattle, there is a predominance of first degree AV blocks [6]. In horses, second degree Mobitz type I episodes are more frequent, and they are characterized by a gradual increase in the length of the $\mathrm{PR}$ segment before an isolated $\mathrm{P}$ wave is identified [8]. In the present investigation, a predominance of second degree AV blocks of Mobitz type II was observed in calves, which represents new information regarding the cardiac rhythm of this species.
Supraventricular ectopic beats are frequent in older bovines, mainly in dairy cows, and are related to the occurrence of atrial fibrillation [1]. This type of arrhythmia is usually caused by increased vagal tone, stress related to hormones, ectopic atrial focus, and volume overload associated with peak lactation [3].

\section{CONCLUSION}

It is possible that the arrhythmias found in the calves in the current study were physiological events. The animals were otherwise healthy, and did not demonstrate any clinical signs of gastrointestinal dysfunction or other disease that might have justified an increase in their vagal tone. In addition, the decrease in the number of rhythm abnormalities in the older calves that were submitted to a second Holter exam supports the hypothesis that supraventricular ectopic beats and second degree AV blocks of Mobitz type II may be normal in calves up to six months old. Nevertheless, more studies are necessary to establish whether there are any particularities in the development of the atrioventricular node of Holstein calves, or if there are some factors associated with an increase in the vagal tone of these animals that may justify the rhythm disturbances observed. It is also necessary to investigate whether these arrhythmias occur only in Holstein calves or if adult cows or other breeds may also be involved.

\section{MANUFACTURERS}

${ }^{1}$ Cardio Sistemas Comercial e Industrial Ltda. São Paulo, SP, Brazil.

${ }^{2}$ DMS Brasil. São Paulo, SP, Brazil.

${ }^{3}$ Graph Pad Software. San Diego, CA, USA. 
Ethical approval. All procedures, treatments and animal care were in compliance with the guidelines of the Comissão de Ética no Uso de Animais - CEUA of the School of Veterinary Medicine and Animal Science, University of São Paulo.
Declaration of interest. The authors report no conflicts of interest. The authors alone are responsible for the content and writing of paper.

\section{REFERENCES}

1 Constable P.D., Muir W.W. III, Bonagura J.D., Rings D.M. \& St. Jean G. 1990. Clinical and electrocardiographic characterization of cattle with atrial premature complexes. Journal of the American Veterinary Medical Association. 9: 1163-1169.

2 Hagen K., Langbein J., Schmied C., Lexer D. \& Waiblinger S. 2005. Heart rate variability in dairy cows-influences of breed and milking system. Physiology \& Behavior. 85(2): 195-204.

3 Machida N., Nakamura T., Kiryu K. \& Kagota K. 1993. Electrocardiographic features and incidence of atrial fibrillation in apparently healthy dairy cows. Journal of Veterinary Medicine Series A. 40: 233-239.

4 Machida N., Okamoto Y., Minami S., Yamaga Y. \& Kagota K. 1991. Cardiac arrhythmias in normal Holstein heifers. Journal of the Japan Veterinary Medical Association. 44: 1176-1179.

5 Mohr E., Langbein J. \& Nürnberg G. 2002. Heart rate variability: a noninvasive approach to measure stress in calves and cows. Physiology \& Behavior. 75(1-2): 251-259.

6 Naghadeh B.D., Dezfouli M.R. \& Rezakhani A. 1998. A survey on frequency of A-V blocks in cattle. Journal of the Faculty of Veterinary Medicine University of Tehran. 54: 97-105.

7 Petrie J. 2005. Practical application of holter monitoring in dogs and cats. Clinical Techniques in Small Animal Practice. 20: 173-181.

8 Reef V.B. 1999. Arrhythmias. In: Marr C.M. (Ed). Cardiology of the Horse. London: W.B. Saunders Co., pp.179-209.

9 Rezakhani A., Oloumi M.M. \& Ahmadi R. 1996. Atrial fibrillation in a cow with fetal maceration. Canadian Veterinary Journal. 37: 625.

10 Rezakhani A., Paphan A.A. \& Gheisari H.R. 2004. Cardiac dysrhythmias in clinically healthy heifers and cows. Revue de Médecine Vétérinaire. 155: 159-162.

11 Scheer P., Svoboda P., Doubek J., Radvanová J., Radvan M. \& Kantorová I. 2005. The electrocardiographic Holter monitoring in experimental veterinary practice. Physiological Research. 59: 59-64. 